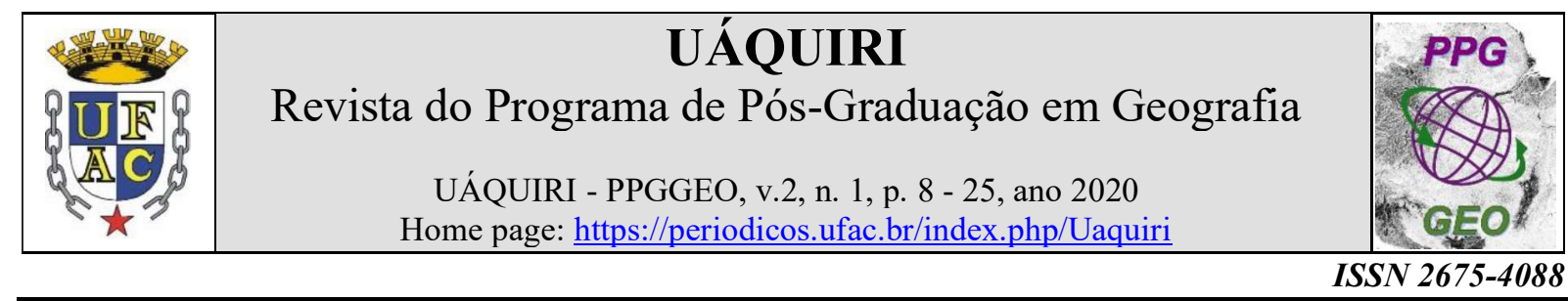

\title{
EXPEDIÇÕES GEOGRÁFICAS DA UFAC: AUTOAVALIAÇÃO E IMPACTOS NA FORMAÇÃO E ATUAÇÃO PROFISSIONAL DO GEÓGRAFO
}

\author{
Msc. Anderson Azevedo Mesquita ${ }^{1,4^{*}}$, Dr. Rodrigo Otávio Peréa Serrano ${ }^{1,3}$, Dr. Waldemir Lima \\ dos Santos ${ }^{1,5}$, Dr. José Genivaldo do Vale Moreira ${ }^{2,3}$ \\ ORCID: http://orcid.org/0000-0003-0947-8070; https://orcid.org/0000-0002-7786-8305; \\ https://orcid.org/0000-0002-5306-5612; https://orcid.org/0000-0002-2994-8482
}

\begin{abstract}
${ }^{1}$ Professor do curso de Geografia da Universidade Federal do Acre (UFAC), Centro de Filosofia e Ciências Humanas, Rio Branco, Acre, Brasil; ${ }^{2}$ Professor da UFAC, Centro Multidisciplinar, Cruzeiro do Sul, Acre, Brasil; ${ }^{3}$ Professor do programa de pós-graduação em Ciência, Inovação e Tecnologia para a Amazônia da UFAC, Rio

Branco, Acre, Brasil; ${ }^{4}$ Doutorando do Programa de pós-graduação mestrado e doutorado em Geografia da Universidade Federal de Rondônia, Porto Velho, Rondônia; ${ }^{5}$ Professor do programa de pós-graduação mestrado em Geografia da UFAC, Rio Branco, Acre.

*amgeoufac@hotmail.com
\end{abstract}

Recebido em: 06/02/2020; Aceito em: 09/04/2020. Publicado em: 22/06/2020.

DOI:

\begin{abstract}
RESUMO
O desenvolvimento de projetos, por lógica requer que um processo de acompanhamento e avaliação seja efetivado. Assim, o presente manuscrito realiza uma discussão sobre o desenvolvimento da segunda expedição geográfica da Universidade Federal do Acre focada no processo de autoavaliação do projeto, e dos seus impactos para os discentes envolvidos na atividade. Para isso, um survey on-line foi desenvolvido com auxílio do Google Forms ${ }^{\circledR}$, e aplicado aos discentes. O survey foi estruturado em seções visando o recolhimento de informações sobre o perfil dos discentes, custos financeiros, contribuição para formação teórico e prática em áreas da geografia, e, planejamento e execução das ações. A síntese quantitativa das respostas foi processada nos softwares Bioestat ${ }^{\circledR}$, Ecxel ${ }^{\circledR}$ e $I R a M u T e Q \circledR$. Por fim, os resultados indicam que os participantes possuem média de idade e renda familiar mensal, respectivamente, de 28 anos e $\mathrm{R} \$ 1552,38$. Para a maioria dos discentes a expedição teve relevante contribuição na formação teórica e prática nas áreas de geografia física, humana e representação do espaço. No quesito planejamento e execução, observa-se o mesmo comportamento positivo na avaliação. Por fim, conclui-se que a expedição geográfica se tornou uma referência positiva para a valorização e ampliação da qualidade dos cursos de geografia da Ufac.
\end{abstract}

Palavras-chave: Avaliação; Projeto; Expedição.

UFAC'S GEOGRAPHICAL EXPEDITIONS: SELF-ASSESSMENT AND IMPACTS ON GEOGRAPHER'S TRAINING AND PROFESSIONAL ACTION

\begin{abstract}
The development of projects by logic requires that a monitoring and evaluation process be carried out. Thus, this chapter discusses the development of the second geographic expedition at the Federal University of Acre focused on the project's self-assessment process, and its impacts on the students involved in the activity. For this, an online survey was developed with the help of Google Forms ${ }^{\circledR}$ and applied to students. The survey was structured in sections aiming to collect information about the students' profile, financial costs, contribution to theoretical and
\end{abstract}


practical training in areas of geography and planning and execution of actions. The quantitative synthesis of the responses was processed using the Bioestat ${ }^{\circledR}$, Ecxel ${ }^{\circledR}$ and IRaMuTeQ ${ }^{\circledR}$ software. Finally, the results indicate that the participants have an average age and monthly family income, respectively, of 28 years and R \$1552.38. For most of the students, the expedition had a relevant contribution to theoretical and practical training in the areas of physical, human geography and space representation. Regarding planning and execution, the same positive behavior is observed in the evaluation. Finally, it is concluded that the geographic expedition has become a positive reference for the enhancement and expansion of the quality of Ufac's geography courses.

Keywords: Evaluation; Project; Expedition.

\section{EXPEDICIONES GEOGRÁFICAS DE UFAC: AUTOEVALUACIÓN E IMPACTOS EN LA FORMACIÓN Y EL DESEMPEÑO PROFESIONAL DO GEOGRAFO}

\section{RESUMEN}

El desarrollo de proyectos por lógica requiere que se lleve a cabo un proceso de monitoreo y evaluación. Por lo tanto, este capítulo analiza el desarrollo de la segunda expedición geográfica en la Universidad Federal de Acre centrada en el proceso de autoevaluación del proyecto y sus impactos en los estudiantes involucrados en la actividad. Para esto, se desarrolló una encuesta en línea con la ayuda de Google Forms ${ }^{\circledR}$ y se aplicó a los estudiantes. La encuesta se estructuró en secciones con el objetivo de recopilar información sobre el perfil de los estudiantes, los costos financieros, la contribución a la capacitación teórica y práctica en áreas de geografía y la planificación y ejecución de acciones. La síntesis cuantitativa de las respuestas se procesó utilizando el software Bioestat ${ }^{\circledR}$, Ecxel ${ }^{\circledR}$ e IRaMuTeQ ${ }^{\circledR}$. Finalmente, los resultados indican que los participantes tienen una edad promedio y un ingreso familiar mensual, respectivamente, de 28 años y R \$ 1552.38. Para la mayoría de los estudiantes, la expedición tuvo una contribución relevante al entrenamiento teórico y práctico en las áreas de representación física, geografía humana y espacio. En cuanto a la planificación y ejecución, se observa el mismo comportamiento positivo en la evaluación. Finalmente, se concluye que la expedición geográfica se ha convertido en una referencia positiva para la mejora y expansión de la calidad de los cursos de geografía de Ufac.

Palabras clave: Evaluación; Proyecto; Expedición

\section{INTRODUÇÃO}

As "expedições geográficas" são atividades integradas de ensino, pesquisa e extensão previstas no currículo acadêmico dos cursos de licenciatura e bacharelado em geografia da Universidade Federal do Acre. No geral, as expedições ocorrem em ciclos bianuais e são programadas e planejadas pelo corpo docente, técnico e discente do curso, e recebe financiamento da administração superior da IES, além de patrocínios de outras instituições parceiras.

As expedições geográficas podem ser consideradas como um evento itinerante onde o corpo docente e discente do curso realizam visitas técnicas em instituições que possuem afinidade com a área de formação do geógrafo. Além das visitas técnicas, o projeto permite a formação qualificada dos discentes uma vez que há possibilidade de integrar o conhecimento teórico com o prático, considerando as diversas atividades desenvolvidas em biomas e realidades geográficas ao longo do Brasil. 
Durante a II Expedição geográfica objetivos foram traçados visando o processo de formação qualificada, interdisciplinar e prática dos discentes dos cursos de geografia da Ufac, além de proporcionar trocas de experiências que fortalecessem o intercâmbio entre instituições e a possibilidade de construção de caminhos e parcerias para o incentivo de formação na área da pós-graduação em geografia. A segunda expedição geográfica ocorreu no ano de 2018, com duração de 25 dias, tendo como destino a cidade de Curitiba - PR.

Neste sentido, ao se traçar metas e objetivos o caminho natural é que um processo de avaliação e autoavaliação seja realizado, afinal tais mecanismos são essenciais e devem ser mantidos para que o sucesso de qualquer projeto seja alcançado. Avaliar é acima de tudo um ato necessário para a busca e consolidação de objetivos e metas, sem avaliação não é possível progredir. A avaliação está presente nos processos mais importantes da vida social e nacional, indicado os caminhos que deverão ser percorridos ou evitados (COTTA, 2016; TARAS, 2010; GRILLO, 2010).

Qualquer processo avaliativo requer do avaliador e do avaliado a disponibilidade, sensibilidade e o entendimento que o produto da avaliação será a busca da qualidade e correção de possíveis equívocos. Assim, a avaliação se transforma em um protocolo ético e emancipatório necessário, independente da variável ou da dimensão física e social abordada. Portanto, a avaliação deverá ser um processo contínuo e integrado a cultura organizacional, política e prática de qualquer instituição, e deve fazer parte da atividade e reflexão diária de todo cidadão (VIEIRA, 2013; OKADA, 2013; FELIX, 2011).

Neste contexto, acreditando no papel e na importância que o ato de avaliar representa, o presente manuscrito intitulado "expedições geográficas da Ufac: autoavaliação e impactos na formação e atuação profissional do geógrafo", objetiva a apresentação de informações e análises quantitativas e qualitativas referentes ao desenvolvimento do projeto, partindo do pressuposto da autoavaliação indicada pelos alunos participantes.

Acredita-se que o feedback demonstrado pelos participantes se tornará em uma importante ferramenta para aperfeiçoamento e ampliação dos impactos positivos do projeto da "Expedição Geográfica". Da mesma forma, a avalição terá uma função social de apresentar para sociedade e comunidade acadêmica, o retorno que o investimento em ações desta natureza podem proporcionar no ganho da qualidade na formação de profissionais, bem como na promoção das ações que são intrínsecas da instituição Universidade, qual seja o de desenvolver o ensino, a pesquisa e a extensão de forma associada e integrada. 


\section{METODOLOGIA}

Para a realização da avaliação da "II Expedição Geográfica", um survey on-line foi desenvolvido com auxílio do Google Forms ${ }^{\circledR}$, e aplicado aos 29 (vinte e nove) discentes que participaram das atividades do projeto. O formulário foi estruturado nas seguintes sessões: identificação do perfil social e econômico dos participantes; estimativas de gastos com hospedagem e alimentação; avaliação da contribuição teórica e prática nas áreas de geografia física, humana e representação do espaço; e, valorização profissional e intercâmbio com instituições. Duas questões abertas foram indicadas na parte final do survey para que os participantes pudessem relatar impressões sobre a contribuição e os pontos positivos e negativos do projeto.

O survey on-line é uma importante ferramenta contemporânea que é amplamente utilizada para a coleta de informações durante o desenvolvimento de pesquisas no âmbito acadêmico e comerciais. O survey tem a vantagem de interagir com o ambiente digital, e proporcionar um rápido alcance quando comparado aos formulários tradicionais. Outra vantagem do survey on-line é a drástica redução de custos e logísticas, fatores importantes em uma realidade que prioriza a otimização de recursos (BABBIE, 1999; AMARO, 2005; MELO, 2010).

Para análise dos dados, os softwares Bioestat ${ }^{\circledR}$, Ecxel ${ }^{\circledR}$ e IRaMuTeQ ${ }^{\circledR}$ foram utilizados para sintetizar informações, indicar relatórios de estatística descritiva e análise de similitude. As questões relacionadas a sessão de identificação do perfil social e econômico dos participantes, foram analisadas a partir de estatística descritiva e elaboração de tabela de frequência. Para as demais sessões, excluindo-se as abertas, foram elaborados gráficos e histogramas. As questões abertas foram analisadas com o uso de relatórios de similitude e "nuvens de palavras".

Para as questões objetivas foi estipulado escala de avaliação de qualidade, considerando, neste cenário, a opinião de cada participante em relação ao impacto do projeto para sua formação. Cada participante obteve acesso ao questionário por meio digital, sendo este amplamente divulgado e incentivado para preenchimento. As escalas de avaliação variavam em 05 (cinco) intensidades, dentre estes, os conceitos: "péssimo", "ruim", "regular", "bom" e "ótimo". Por fim, compreende-se como avaliação "positiva" respostas acumuladas nos conceitos "bom e ótimo", e, como avaliação "negativa" respostas acumuladas nos conceitos "péssimo, regular e ruim". 


\section{RESULTADOS E DISCUSSÃO}

\subsection{Do perfil dos participantes}

Considerando a análise do perfil dos participantes observa-se que 55,17\% eram do sexo masculino e 44,83\% feminino, conforme indicado na figura 01 . Pelos dados constata-se uma maior procura por participação na expedição de discentes do sexo masculino, no entanto, tal fato deve ser considerando a partir da proporcionalidade de alunos matriculados nos cursos de acordo com o sexo. Contudo, ressalta-se que a diferença percebida não pode ser visualizada como de alta amplitude dada a relativa diferença de 10,34\%.

Figura 01: (\%) do sexo dos participantes

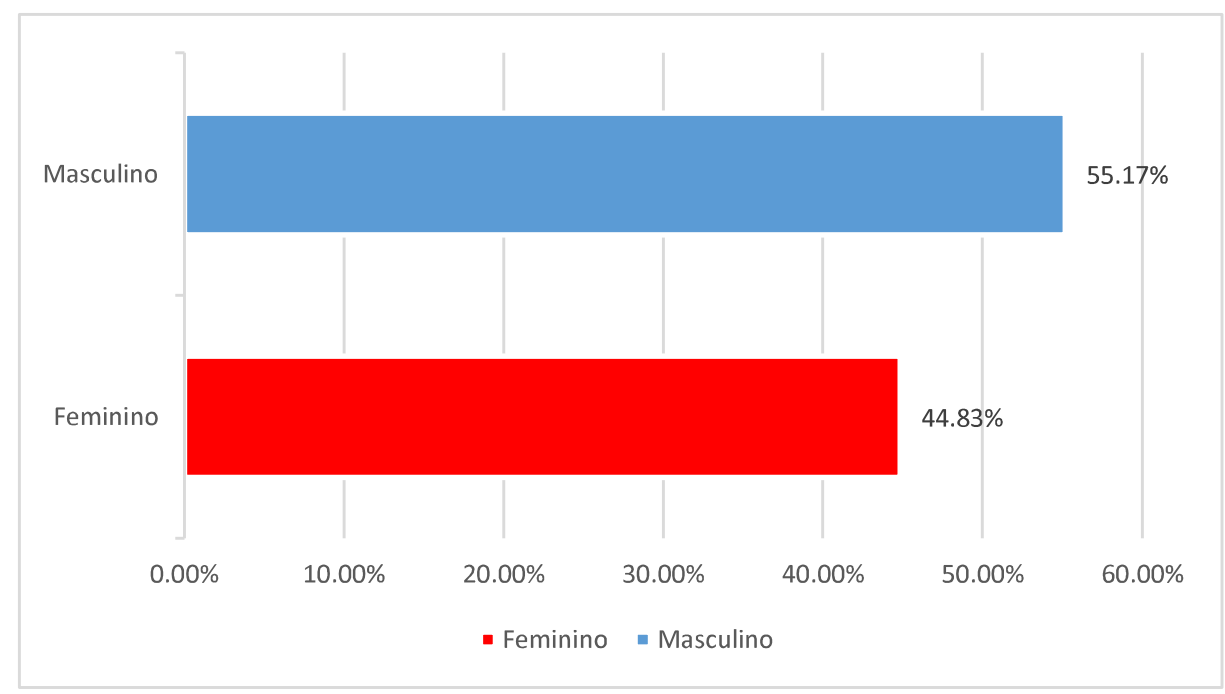

Em relação ao período cursado observa-se que 76\% estavam matriculados nos períodos finais de seus respectivos cursos, e o restante aproximadamente $34 \%$, estavam com matrículas ativas entre o $4^{\circ}$ e $6^{\circ}$ período, conforme apresentado na figura 02 . Destaca-se que a participação de discentes dos períodos iniciais não foi incentivada durante o processo seletivo para composição do projeto. Tal fato se justifica em virtude da menor integralidade de créditos teóricos e práticos destes alunos na estrutura curricular do curso, assim como da menor possibilidade de participação no projeto dos alunos concludentes. 
Figura 02: (\%) de alunos participantes por período cursado

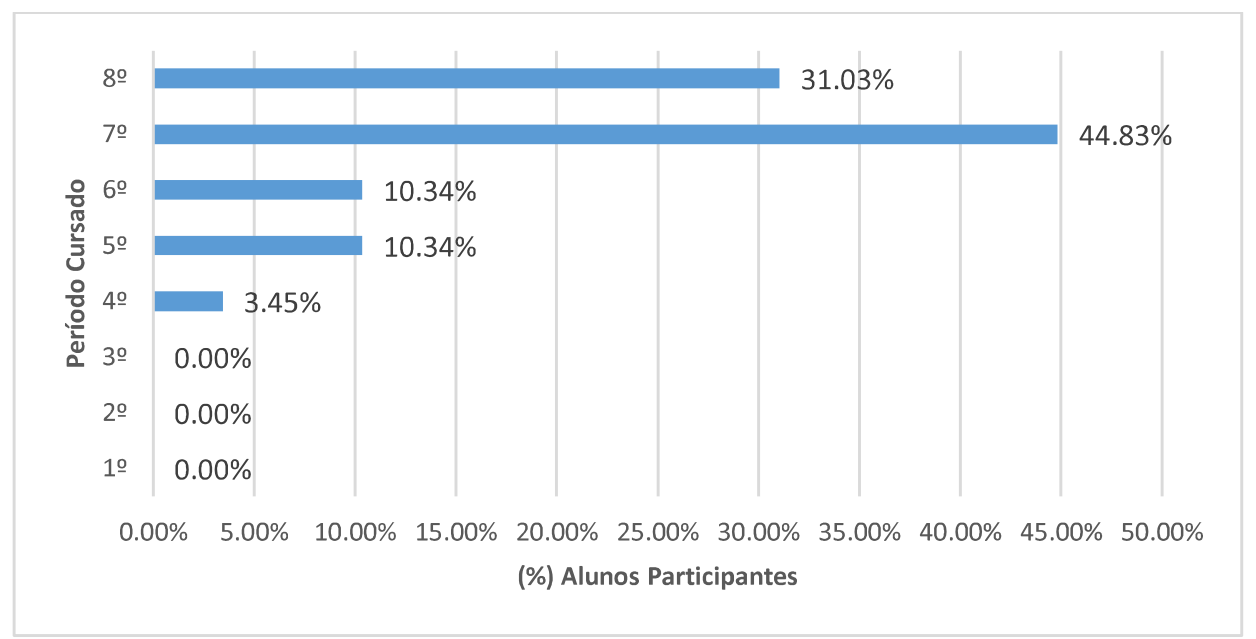

De acordo com a figura 03 , aproximadamente $80 \%$ do discentes estavam cursando licenciatura em geografia e apenas $20 \%$ cursavam bacharelado, o que indica uma elevada concentração dos participantes na modalidade de licenciados. Contudo, deve-se destacar mais uma vez a proporcionalidade de matrículas nos cursos, em especial, na licenciatura, que durante o período de seleção estava com três cursos em funcionamento, considerando além da sede em Rio Branco-AC, os programas especiais de interiorização nos municípios de Cruzeiro do Sul - AC e Sena Madureira-AC.

Figura 03: (\%) de alunos participantes por modalidade no curso

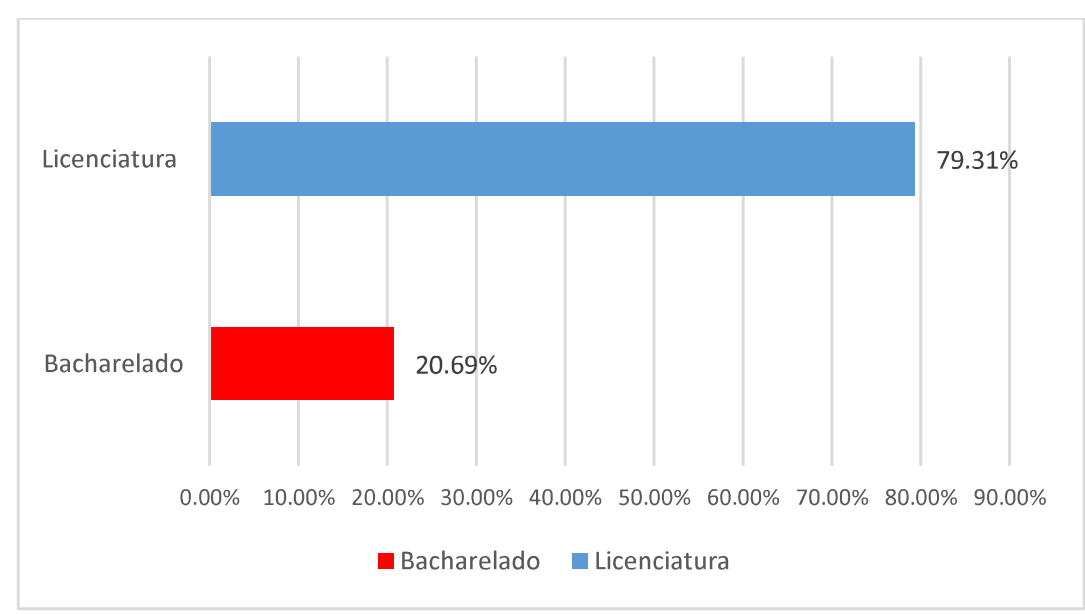

Em relação a idade, a tabela 01 indica que aproximadamente $56 \%$ dos discentes estão na faixa etária de 20 a 24 anos. Portanto, a maioria dos participantes são considerados jovens adultos o que implica um grande desafio para a organização da atividade. No entanto, mesmo com o maior percentual de jovens adultos observa-se uma frequência de idade importante entre 
as faixas de 35 a 44 anos. Por fim, a média de idade dos foi calculada em aproximadamente 28 anos, a mediana em 23 , com um coeficiente de variação de $29 \%$.

Tabela 1: (\%) distribuição de frequência de idade dos participantes

$\begin{array}{lccc}\text { Classes } & \mathbf{X i} & \mathbf{F i} & \text { Percentual } \\ 20|-| 24 & 22 & 16 & 55,17 \% \\ 25|-| 29 & 27 & 5 & 17,24 \% \\ 30|-| 34 & 32 & 1 & 3,45 \% \\ 35|-| 39 & 37 & 3 & 10,34 \% \\ 40|-| 44 & 42 & 4 & 13,79 \% \\ \text { Total } & & \mathbf{2 9} & \mathbf{1 0 0 \%}\end{array}$

Em um curso com reconhecido perfil socioeconômico de baixa renda dos seus alunos, a participação no projeto da expedição geográfica, infelizmente ainda pode ser determinada em função da capacidade de custeio durante os mais de 20 dias de desenvolvimento das ações. Neste sentido, a tabela 02 indica que aproximadamente $62 \%$ dos discentes possuem renda familiar média de até R\$1527, ou seja, aproximadamente 1,5 salário mínimo. Para estes alunos, o auxílio financeiro fornecido pela universidade, e o apoio levantado a partir de doações, rifas e vendas foram determinantes para a participação no projeto. Por fim, a renda média familiar calculada é de $\mathrm{R} \$ 1522,40$, a mediana em $\mathrm{R} \$ 1000,00$, com um elevado coeficiente de variação de $66,33 \%$.

Tabela 2: (\%) distribuição de frequência de idade dos participantes

$\begin{array}{cccc}\text { Classes } & \mathbf{X i} & \mathbf{F i} & \text { Percentual } \\ 146|-| 836 & 491 & 6 & 20,69 \% \\ 837|-| 1527 & 1182 & 12 & 41,38 \% \\ 1528|-| 2218 & 1873 & 3 & 10,34 \% \\ 2219|-| 2909 & 2564 & 2 & 6,90 \% \\ 2910|-| 3600 & 3255 & 6 & 20,69 \% \\ \text { Total } & & \mathbf{2 9} & \mathbf{1 0 0 \%}\end{array}$

Conforme destacado, a capacidade de custeio dos discentes pode ser considerada o principal desafio para realização da expedição, o que implica que o auxílio financeiro fornecido pela universidade é uma condição elementar apontada pelos discentes para sua respectiva participação no projeto. A figura 04 representa que 93\% dos discentes destacaram no questionário que o auxílio financeiro foi determinante para sua participação no projeto. Neste 
sentido, observa-se que a instituição possui uma grande responsabilidade social quando considerada a capacidade de financiamento de atividades desta natureza.

Figura 04: (\%) de alunos que indicaram que o auxílio financeiro fornecido pela IES foi determinante para participação no projeto

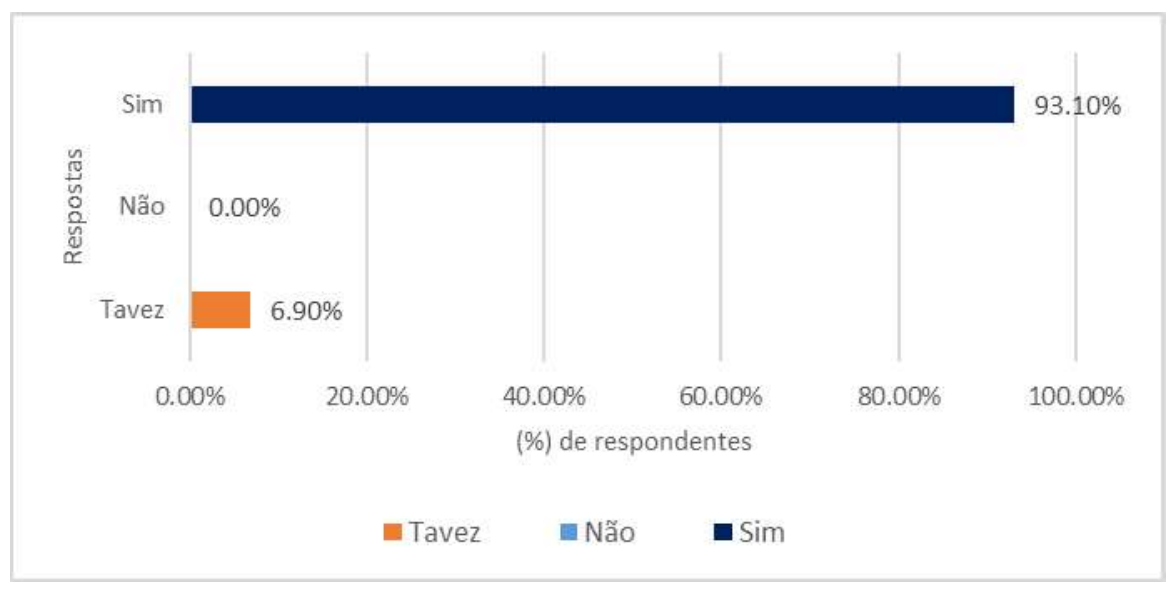

Concluindo as análises referentes ao perfil dos participantes, referente ao gasto diário com alimentação e hospedagem observa-se que para alimentação $86,02 \%$ gastaram $\mathrm{R} \$ 15,00$ até $\mathrm{R} \$ 44,00$, sendo a média diária de $\mathrm{R} \$ 33,41$, mediana de $\mathrm{R} \$ 30,00$, e o coeficiente de variação de 34,30\%. Para hospedagem 82,76\% dos participantes gastaram R \$ 40,00 até R\$ 51,00 , sendo a média diária de $\mathrm{R} \$ 50,52$, mediana de $\mathrm{R} \$ 50,00$, e o coeficiente de variação de $15,73 \%$.

É importante destacar que o impacto diário de custos na alimentação pode ter sido menor, em função da mobilização dos discentes que buscaram doações de mantimentos no período de planejamento do projeto, e mesmo pela oferta de café da manhã nos hotéis durante as hospedagens. Outro condicionante é que durante os translado a equipe de planejamento negociava os preços da alimentação e da hospedagem visando valores abaixo do mercado.

\subsection{Contribuição teórica e prática}

Um dos maiores desafios dentro da esfera educacional superior é proporcionar uma formação qualificada e integrada entre o universo teórico e prático das estruturas curriculares dos cursos de graduação (KUENZER, 2018; GONDIM, 2002). O sucesso do profissional formado, passa obrigatoriamente, pela sua capacidade assimilativa e reflexiva em relação aos conceitos e leis gerais típicas da sua área de formação. No entanto, é a sua capacidade interventiva e cotidiana na proposição de soluções e resolução de problemas, que em último 
caso, define sua ascensão e estabilização enquanto referência profissional (VIEIRA, 2006; CHIAVENATO, 2005).

Seguindo esta lógica vários estudos (COMPIANI, 1993; BONITO, 1997) indicam a relevância e o impacto positivo do desenvolvimento de atividades práticas de campo para a formação em nível superior. Na ciência geográfica este impacto tende a ser ainda mais relevante, em virtude de seu objeto de estudo, qual seja de compreender a formação do espaço a partir da articulação e integração do social com o físico (BUENO, 2009; SUERTEGARAY, 2009; 2015). Portanto, considerando esta especificidade na geografia, a expedição tem como meta elementar proporcionar ao discentes experiências formativas diferenciadas com forte impacto na sua futura prática profissional.

Buscando avaliar o impacto da expedição no processo formativo dos discentes, foram inseridas 06 (seis) questões referentes a contribuição do projeto para a formação teórica e prática nas áreas da geografia física, humana e de representação do espaço. Por fim, conforme indicado na metodologia, para cada questão foi atribuída uma escala de qualidade que variava de "péssimo" a "ótimo", sendo a intepretação de avaliação positiva as indicações de avaliação "ótimo" e "bom", e como avaliação negativa as indicações "péssimo", "ruim" e "regular".

Ao avaliar a "contribuição da II expedição geográfica para a formação teórica nas disciplinas da geografia humana", a figura 05, apresenta que 86,21 \% dos participantes indicaram avaliação nas escalas "ótimo e bom", 13,79\% "regular", e 0\% "ruim e péssimo". Tal resultado traduz, na opinião dos participantes, que a expedição teve um forte impacto positivo para a formação teórica na área da geografia humana, fato este equivalente a avaliação indicada, conforme figura 06, para a "contribuição na formação prática", onde 89,65 \% dos respondentes indicaram avaliação "ótimo e bom", 6,90\% "regular", 3,45 "ruim", e 0\% "péssimo".

Figura 05: (\%) avaliação da contribuição da II expedição geográfica para a formação teórica na área de geografia humana

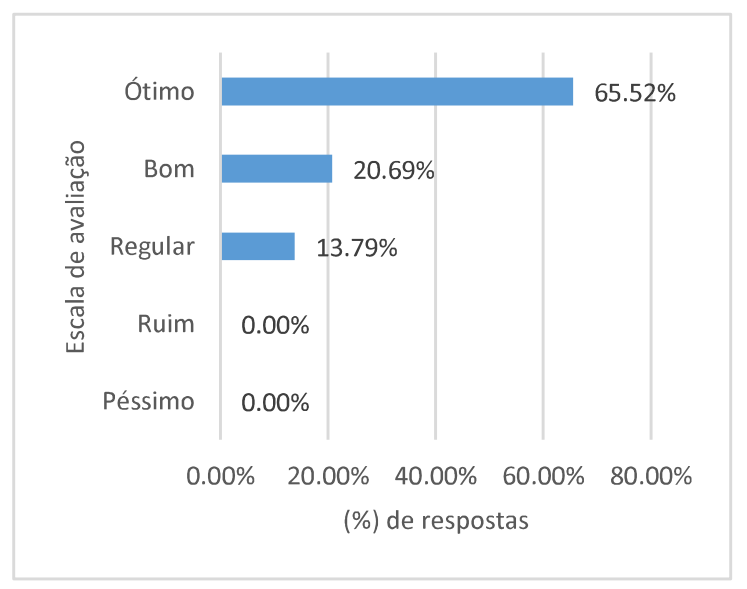

Figura 06: (\%) avaliação da contribuição da II expedição geográfica para a formação prática na área de geografia humana

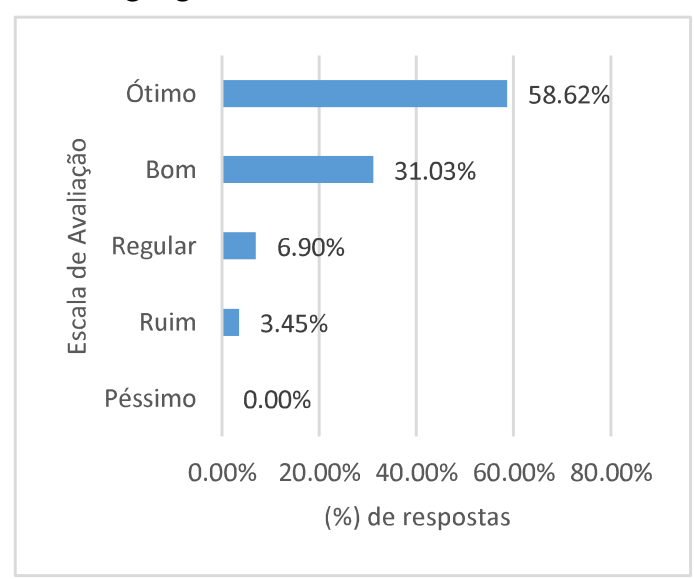


Ao avaliar a "contribuição da II expedição geográfica para a formação teórica nas disciplinas da geografia física", a figura 07, apresenta que 100,00 \% dos participantes indicaram avaliação na escala "ótimo". Tal resultado é surpreendente e traduz, na opinião dos participantes, que a expedição é elementar, e fortemente impactante para a qualidade formativa na área da geografia física.

A figura 08 , representa o impacto da expedição na "contribuição na formação prática na área de geografia física”. Neste sentido, o comportamento dos respondentes também indicou uma avaliação fortemente positiva, pois $100 \%$ avaliaram a questão nas escalas "ótimo e bom". O fato de não existir nenhuma avaliação considerada negativa em relação a questão, indica que de forma unânime os participantes concordaram positivamente com a contribuição da expedição neste quesito.

Dado o isolamento geográfico e as diversas dificuldades e desafios materializados em função desta característica, os participantes vislumbraram no desenvolvimento da expedição uma oportunidade única em manter contato com realidades que dificilmente poderiam ser vivenciadas pelos demais discentes dos cursos de geografia da Ufac, especialmente na área de geografia física.

Afinal, a possibilidade de manter contato com outros biomas, ecossistemas, condições climáticas, de relevo, geologia e vegetação proporcionaram ao participantes a junção efetiva e integradora entre o conhecimento teórico e prático tão almejado no âmbito da formação geográfica, afinal tais experiências e vivências, dada as dificuldades proporcionadas pelo isolamento geográfico da nossa região, dificilmente poderiam ser superadas sem a realização de um projeto como o da expedição.

Figura 07: (\%) avaliação da contribuição da II expedição geográfica para a formação teórica na área de geografia física

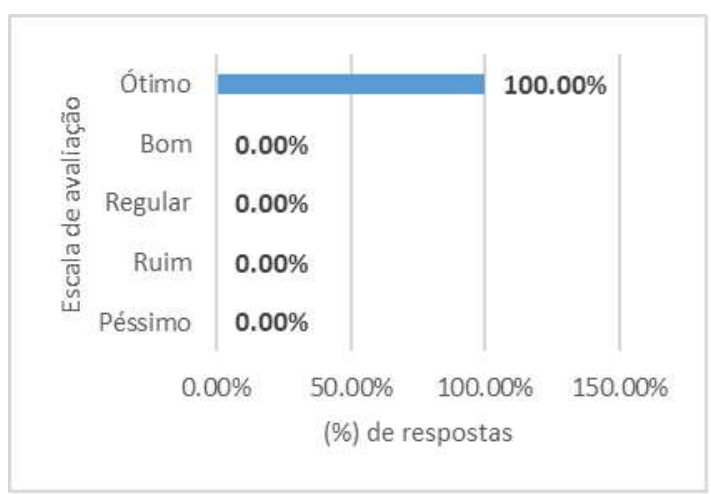

Figura 08: (\%) avaliação da contribuição da II expedição geográfica para a formação prática na área de geografia física

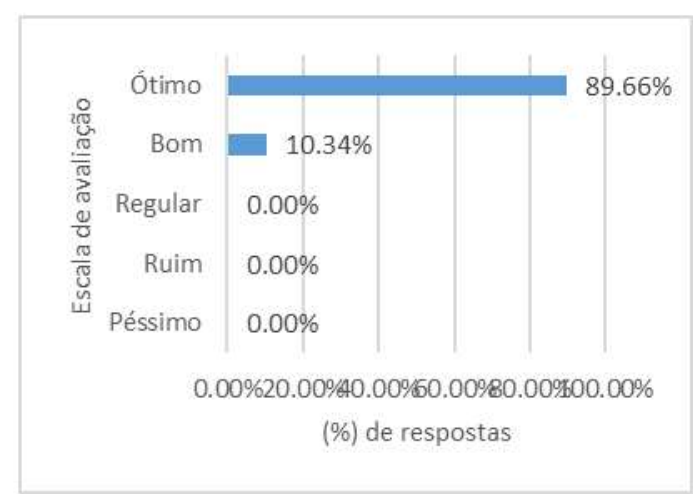


Ao avaliar a "contribuição da II expedição geográfica para a formação teórica nas disciplinas da área de representação do espaço", a figura 09, apresenta que 82,76 \% dos participantes indicaram avaliação nas escalas "ótimo e bom", 6,90\% "regular", e 3,45\% "ruim" e 6,90\% péssimo". Tal resultado traduz, na opinião dos participantes, que a expedição teve um impacto positivo para a formação teórica na área de representação do espaço, no entanto, quando os dados são comparados as duas outras áreas (física e humana), observa-se uma "tímida crítica" de 17,25\% dos participantes que avaliaram a contribuição como "regular", "ruim" e "péssimo".

Figura 09: (\%) avaliação da contribuição da II expedição geográfica para a formação teórica na área de representação do espaço.

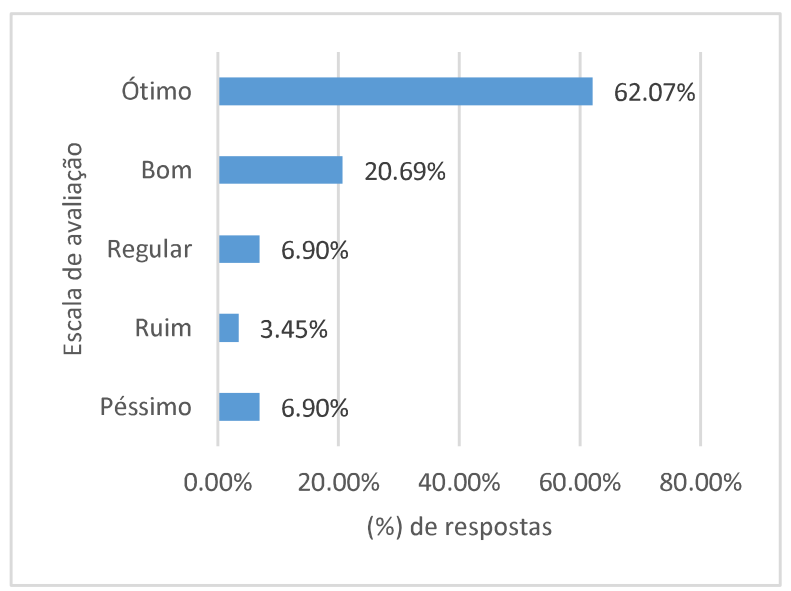

Figura 10: (\%) avaliação da contribuição da II expedição geográfica para a formação prática na área de representação do espaço.

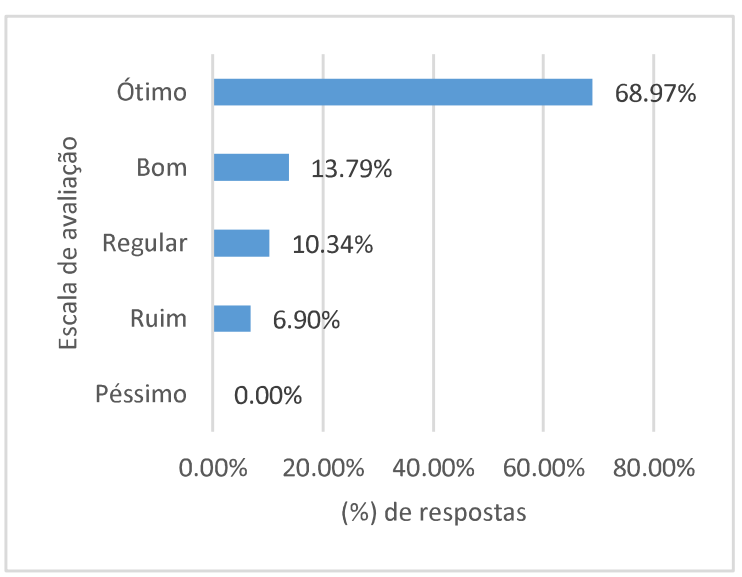

Por fim, embora o resultado tenha se demonstrado positivo, acredita-se que para o desenvolvimento da próxima expedição geográfica, a equipe de coordenação deverá considerar possíveis intervenções visando a ampliação do rendimento dos participantes nesta área tão importante para a prática geográfica.

\subsection{Planejamento, valorização profissional e intercâmbio}

A avaliação do planejamento e execução das ações do projeto proporcionam a equipe organizadora um feedback importante para a correção de possíveis falhas, bem como a ampliação dos procedimentos considerados exitosos. Nesse sentido a figura 11, demonstra que para 100\% dos participantes, sendo manifestado 68,97\% de conceito "ótimo" e 31,03\% de conceito "bom", a execução e planejamento da expedição atingiram aos objetivos propostos no projeto. Tal retorno apresenta um cenário extremamente positivo, pois acredita-se que os 
objetivos e metas foram alcançadas com êxito, mesmo considerando todas as dificuldades de planejamento e execução enfrentadas.

Dento desta dimensão outro ponto relevante se refere a avaliação manifestada pelos discentes quanto a "importância da expedição para a valorização da formação do profissional em geografia". Ressalta-se que um dos objetivos centrais do projeto visava o aumento da "auto estima" dos alunos do curso, dando a estes uma possibilidade formativa diferenciada, e enfrentando os resultados negativos sofridos pelo curso de bacharelado em geografia na última avaliação institucional.

Neste quesito, conforme indicado na figura 12, para 96,55\% dos participantes a avaliação foi "ótimo" e 3,45\% "bom", totalizando $100 \%$ de conceito positivo de avaliação. Tais resultados indicam que os alunos enxergam na expedição uma importante engrenagem de desenvolvimento e valorização da formação do profissional em geografia.

Figura 11: (\%) avaliação do planejamento e execução da expedição considerando os objetivos e metas do projeto.

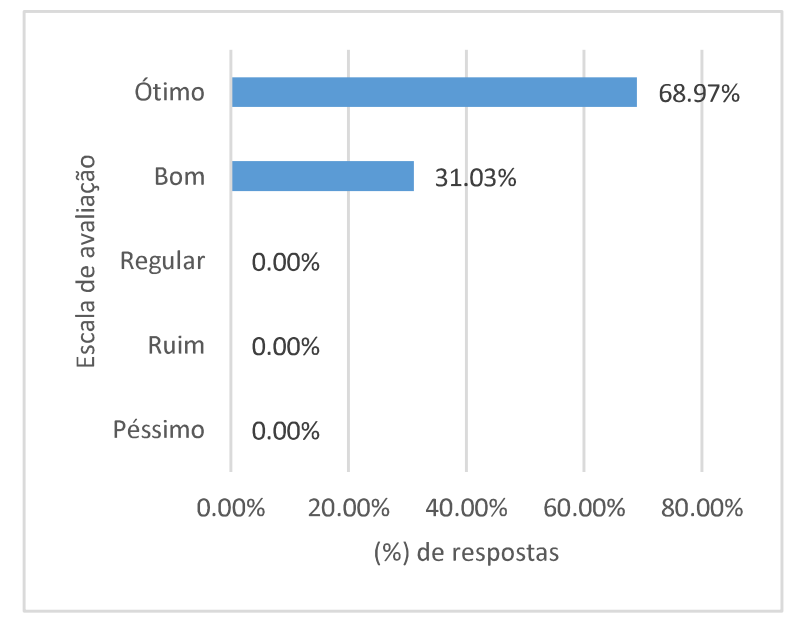

Figura 12: (\%) avaliação da importância da expedição para a valorização da formação do profissional em geografia.

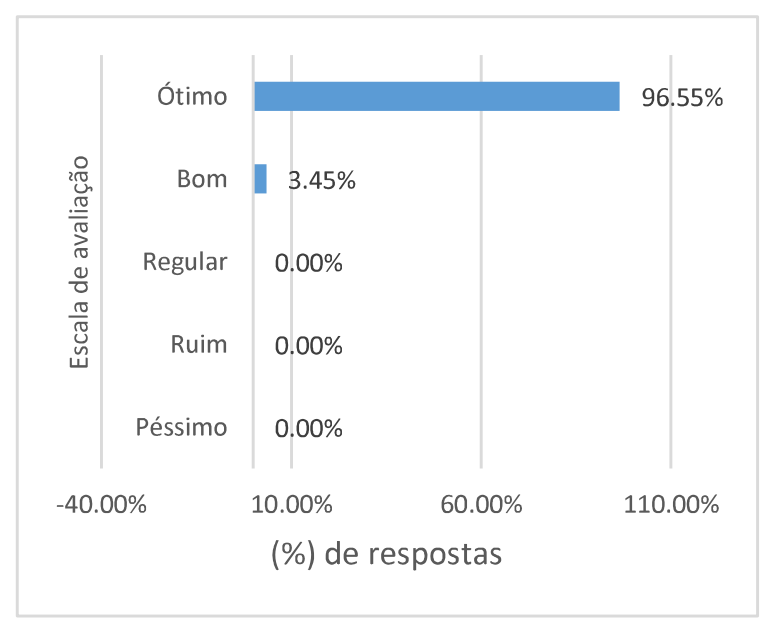

Para além de fomentar a valorização da formação profissional na geografia, a expedição geográfica caracterizava-se como um projeto complexo e completo que mantinha objetivos centrados no desenvolvimento do ensino, da pesquisa e da extensão, elo fundamental e indissociável da universidade. Dentro destes objetivos vislumbrava-se a construção de políticas de intercâmbios institucionais que foram sumariamente realizadas ao longo do desenvolvimento do projeto. Tais intercâmbios foram realizados com instituições e órgãos de reconhecida relevância científica e técnica, tais como: hidrelétricas (Santo Antônio; Itaipu), universidades (Universidade de Rondônia; Universidade Federal de Mato Grosso do Sul; 
Universidade Estadual de Ponta Grossa; Universidade Federal do Paraná); e órgãos de proteção ao meio ambiente (ICMBio).

Considerando as atividades de intercambio desenvolvidas com outras instituições no âmbito do projeto, a figura 13 demonstra que os participantes manifestaram $93,10 \%$ de avaliação no conceito "ótimo", e 6,90\% de avaliação no conceito "bom", totalizando desta forma $100 \%$ de avaliação considerada positiva. Esta realidade indica que a expedição geográfica proporcionou através do intercâmbio com outras instituições, um conjunto de experiências e vivências importantes para a formação acadêmica dos discentes. Outro ponto a se destacar é que a manutenção da rotina de desenvolvimento do projeto poderá proporcionar acordos futuros na área de estágio e até mesmo pós-graduação.

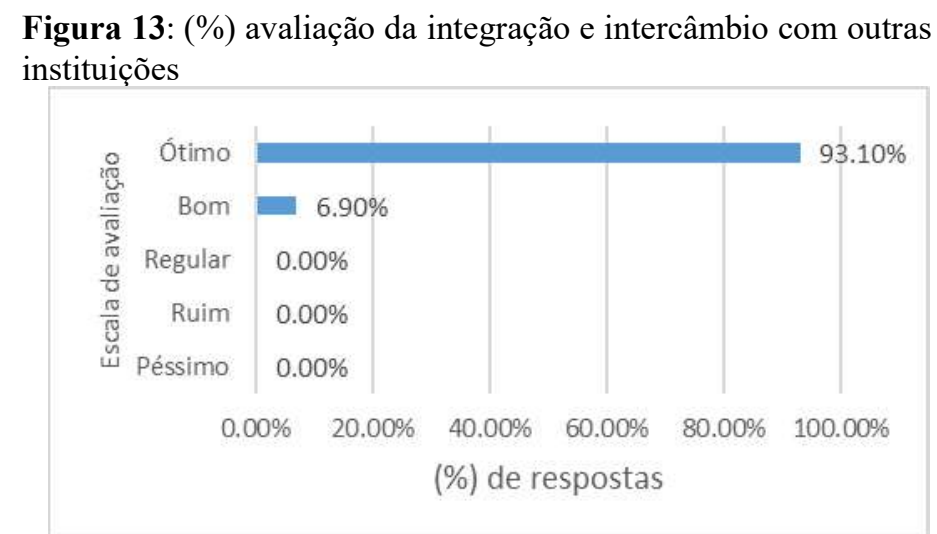

\subsection{Avaliação geral e análise de similitude}

Conforme indicado na metodologia, no survey on-line foram inseridas duas questões abertas, sem escalas de avaliação, para que os alunos discorressem livremente sobre impressões gerais relacionadas a expedição. Nas questões, apenas foi direcionado que as impressões deveriam ser redigidas em função da "importância da expedição geográfica para a formação dos discentes do curso", e "avaliação geral do projeto destacando pontos positivo e negativos".

Para síntese das repostas utilizou-se a análise de similitude do software IRaMuTeQ ${ }^{\circledR}$. A análise de similitude fundamenta-se na teoria dos grafos, que permite a identificação de ocorrência entre palavras, bem como as possíveis conexões existentes entre elas. Neste contexto, é possível identificar conexões entre a estruturas e frequência das palavras dos respondentes refletindo desta forma na compreensão de discursos convergentes e divergentes sobre determinado assunto (PEREIRA, 2018; CAMARGO, 2013; JUSTO, 2014). 
A figura 14, representa a projeção gráfica da análise de similitude elaborada a partir das respostas dos participantes. Ao analisar as conexões entre os termos, observa-se como ponto central, que os participantes destacaram a relevância da expedição considerando a frequência e a conexão central das expressões "importância", "vivenciar", "conhecimento" e "prático". Tais expressões se conectam com as outras três estruturas, sendo que na primeira se destacam os termos "geografia", "essencial" e "formação"; na segunda as expressões "expedição", "profissional"; e na terceira, as expressões "aula", "campo" e "lugar".

Figura 14: estrutura de expressões da análise de similitude referente a avaliação da importância da expedição para a formação dos discentes do curso

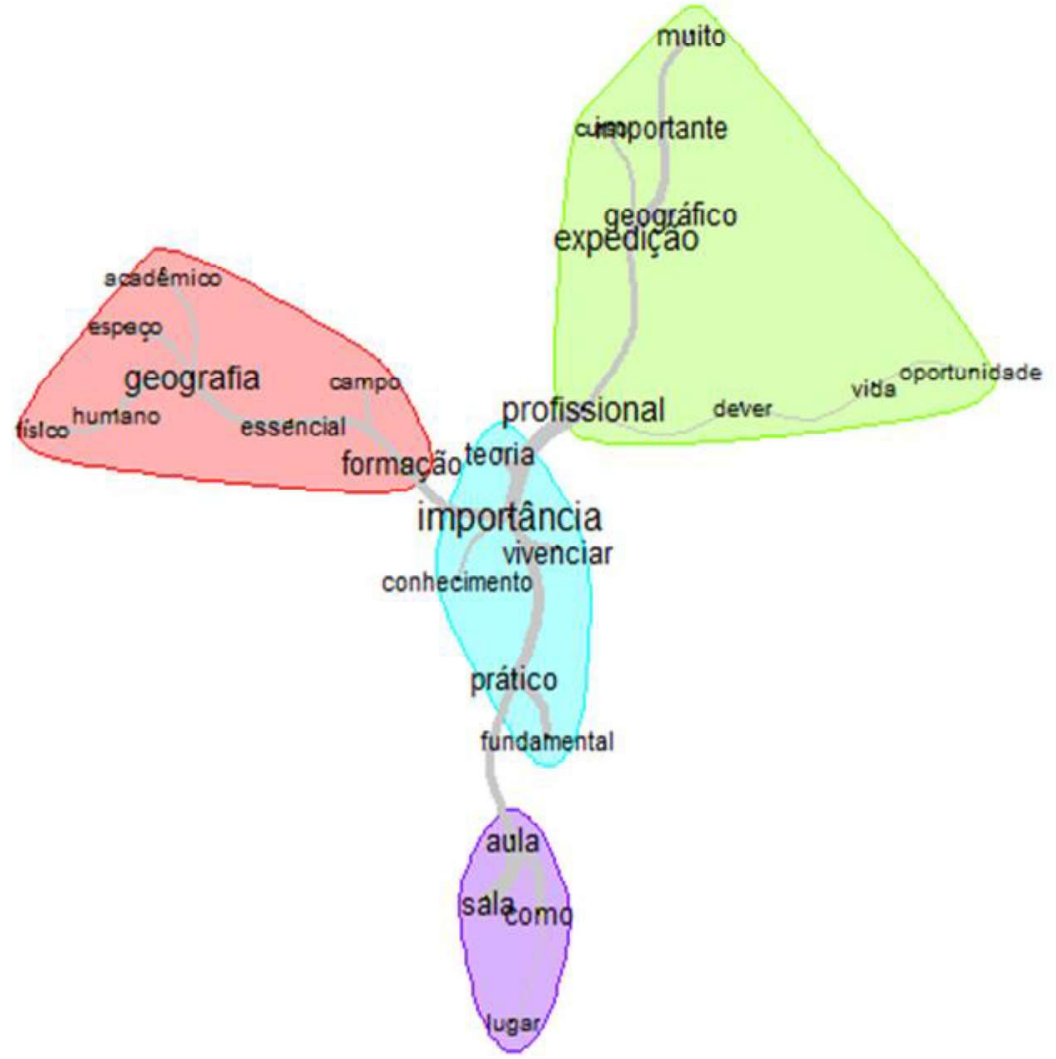

Ainda considerando a análise da figura 14, observa-se na estrutura central que a expedição proporcionou vivências de oportunidades práticas e teóricas importantes através da análise dos discursos dos participantes. Estas oportunidades se conectam com a expedição enquanto um "laboratório de aula" itinerante (terceira estrutura), bem como se conectam com a primeira e segunda estrutura, ressaltando respectivamente, a formação plural (humana, física) e a contribuição da expedição para a formação profissional.

Por fim, ao considerar a análise de similitude verifica-se que a expedição é um projeto de relevante importância para a formação profissional dos participantes, pois agrega em um 
ambiente de "sala de aula itinerante" oportunidades de formação teóricas e práticas que dificilmente poderiam ser vivenciadas no âmbito da formação curricular comum.

Outra avaliação realizada se refere a formação de "nuvens de palavras" a partir da frequência de expressões relatadas na avaliação dos participantes. Logo, a figura 15, demonstra que as expressões centrais destacadas são: "importância"; "profissional"; "expedição"; "geografia"; "aula"; "prático"; "conhecimento"; "geográfico"; "vivenciar"; e "formação". Existem também alguns termos que foram apresentados de forma secundária na nuvem, mas que da mesma forma demonstram um sentido positivo na avaliação dos participantes.

Ao considerar a expressão com maior destaque ou frequência, observa-se três palavras: "expedição", "importância" e "geografia", dentre estas a de maior destaque foi a expressão "importância". Isto demonstra que reconhecidamente os discentes percebem o impacto positivo do desenvolvimento do projeto para a geografia, afinal as três expressões principais expressam exatamente este sentido, ou seja, "A expedição é importante para a geografia".

Por fim, as figuras 16 e 17, representam respectivamente a manifestação dos participantes quanto ao destaque sobre pontos negativos e positivos identificados no projeto. Logo na figura 16, observa-se que os participantes destacaram como pontos negativos as dificuldades encontradas para obtenção de custeio, bem como a aquisição e liberação do ônibus para a realização do projeto.

Figura 15: nuvens de palavras da análise de similitude referente a avaliação da importância da expedição para a formação dos discentes do curso.

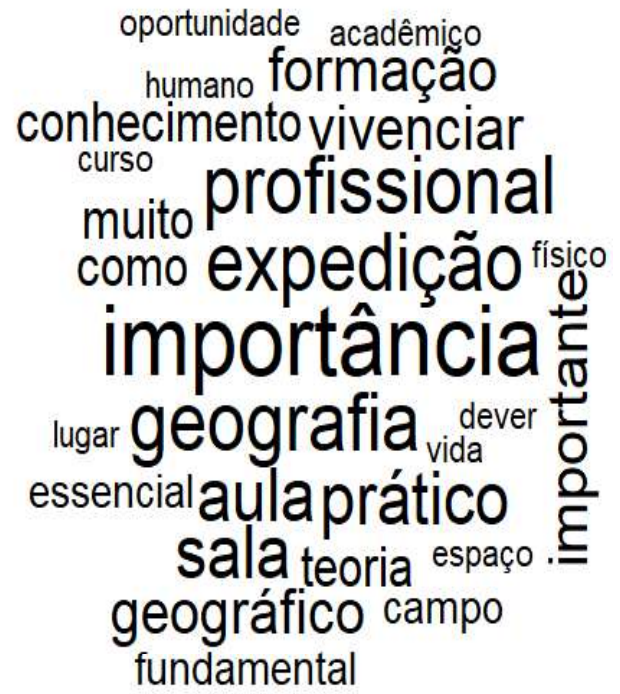

Figura 16: estrutura de expressões da análise de similitude referente a indicação de pontos negativos sobre a expedição

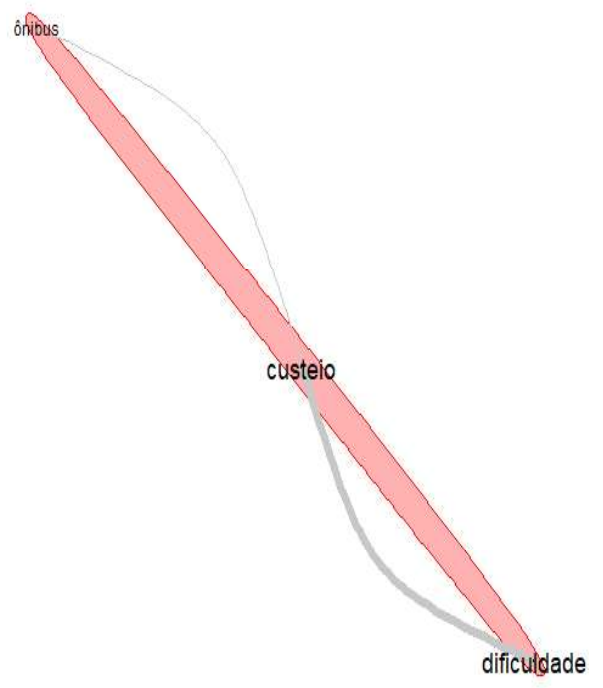


Na figura 17, os discentes indicam como "pontos positivos" a ótima relação entre alunos e os professores (primeira estrutura), a possibilidade dos alunos em adquirir novos conhecimentos (segunda estrutura), e a integração positiva proporcionada pela expedição em relação as visitas realizadas a outras instituições.

Figura 17: estrutura de expressões da análise de similitude referente a indicação de pontos positivos sobre a expedição

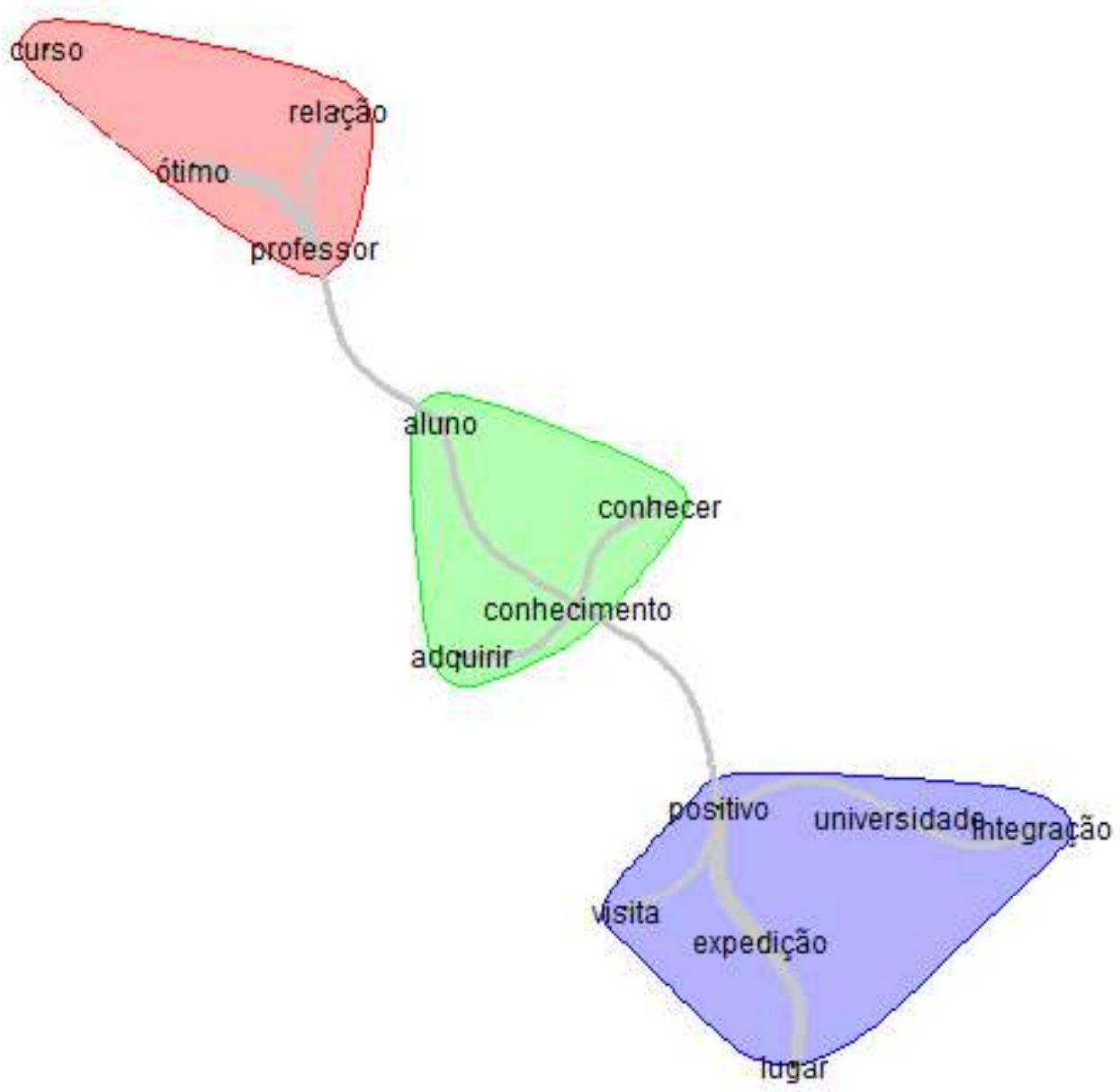

\section{CONCLUSÃO}

Os resultados da avaliação e autoavaliação indicam que a expedição geográfica foi relevante e impactante positivamente para a formação teórica e prática nas áreas de geografia física, humana e representação do espaço, tendo maior contribuição na área de geografia física. Observou-se que o intercâmbio com outros órgãos e instituições foram destacados como experiências importantes nos discursos dos alunos. 
Quanto ao perfil dos participantes observa-se que a maioria só participou da expedição por conta do auxílio financeiro fornecido pela IES. Verificou-se relativa variação na idade e na renda média familiar do grupo, e na descrição dos gastos com hospedagem e alimentação durante o projeto.

Por fim, observa-se quase que por unanimidade entre os participantes que a expedição proporcionou oportunidades positivas para melhor formação dos discentes, sendo inclusive destacado o impacto para a melhora da autoestima e da motivação dos discentes no curso.

\section{AGRADECIMENTOS}

A gestão superior da Universidade Federal do Acre, pelo apoio logístico e acadêmico durante a realização do projeto. Em especial agradecemos aos participantes da expedição que responderam aos survey-online, e que foram fundamentais para a realização deste trabalho.

\section{REFERÊNCIAS}

AMARO, A.; PÓVOA, A.; MACEDO, L. A arte de fazer questionários. Porto, Portugal: Faculdade de Ciências da Universidade do Porto, 2005.

BABBIE, E. Métodos de pesquisas de survey. Belo Horizonte: Ed. da UFMG, 1999.

BONITO, J.; SOUSA, M. Actividades práticas de campo em Geociências: uma proposta alternativa. 1997.

BUENO, M. A. A importância do estudo do meio na prática de ensino em geografia física. 2009.

CHIAVENATO, I. Comportamento organizacional: a dinâmica do sucesso das organizações. Editora Manole, 2005.

COMPIANI, M.; CARNEIRO, C. D. R. Os papéis didáticos das excursões geológicas. Enseñanza de las Ciencias de la Tierra, 1993, 1.2: 90-97.

COTTA, R. M. M.; COSTA, G. D. da. Instrumento de avaliação e autoavaliação do portfólio reflexivo: uma construção teórico-conceitual. Interface-Comunicação, Saúde, Educação, 2016, 20: p. 171-183.

FELIX, G. T.; G., Simone Freitas da Silva. Gestão, avaliação e participação na autoavaliação da UFSM: em busca de compreensões sob distintos horizontes. TRABALHOS $\mathbf{2 5}^{\circ}$ SIMPÓSIO BRASILEIRO, $\mathbf{2}^{\mathbf{0}}, 2011$. 
GONDIM, S. M. G. Perfil profissional e mercado de trabalho: relação com formação acadêmica pela perspectiva de estudantes universitários. 2002.

GRILlO, M. C.; DE FREITAS, A. L. S. AUTOAVALIAÇÃO: por que e como realizála? Por que falar ainda em avaliação? 2010, 45.

KUENZER, A. Z. Competência como práxis: os dilemas da relação entre teoria e prática na educação dos trabalhadores. Boletim técnico do SENAC, 2018, 29.1: 16-27.

MELO, C. de O.; FERREIRA, G. R. M. Adoção de métodos ágeis em uma Instituição Pública de grande porte-um estudo de caso. In: WORKSHOP BRASILEIRO DE MÉTODOS ÁGEIS (AGILE BRASIL 2010). (Anais) Porto Alegre: AgilCoop-Cooperativa de Desenvolvimento Ágil de Software. 2010.

OKADA, A.; MEISTER, I.; BARROS, D. M. V. Refletindo sobre avaliação na era da coaprendizagem e co-investigação. 2013.

PEREIRA, C. Análise de dados qualitativos aplicados às representações sociais. Psicologia, Lisboa, $\quad$ v. 15, n. 1, p. 177-204, jan. 2001. Disponível em http://www.scielo.mec.pt/scielo.php?script $=$ sci arttext\&pid $=$ S087420492001000100008\&lng=pt\&nrm=iso. Acesso em: 13 dez. 2018.

SUERTEGARAY, D. M. A. Pesquisa de campo em geografia. GEOgraphia, 2009, 4.7: 64-68 SUERTEGARAY, D. M. A.; NUNES, J. O. R. A natureza da Geografia Física na Geografia. Terra Livre, 2015, 2.17: 11-24.

TARAS, M. De volta ao básico: definições e processos de avaliação. Práxis Educativa, 2010, 5.2: p. $123-130$

VIEIRA, D.; COIMBRA, J. L. Sucesso na transição escola-trabalho: a percepção de finalistas do ensino superior português. Revista brasileira de orientação profissional, 2006, 7.1.

VIEIRA, I. M. A. A autoavaliação como instrumento de regulação da aprendizagem. 2013. PhD Thesis.

VIZEU C.; BRIGIDO, J., A. M. IRAMUTEQ: Um Software Gratuito para Análise de Dados Textuais. Temas em Psicologia [en linea] 2013, 21 (Diciembre-Sin mes): [Fecha de consulta: 13 de diciembre de 2018. Disponível em: http://www.redalyc.org/articulo.oa?id=513751532016 